\title{
Cardiac transplantation
}

\author{
HARRY M. WINDSOR \\ M.S., F.R.C.S., F.R.A.C.S., F.A.C.S. \\ Cardiac Unit, St Vincent's Hospital, Sydney
}

The validity of many fundamental conceptions has been challenged by the present social and technical revolution. Medicine has not escaped and nothing so exemplifies this as the problem of heart transplants.

The physician over the centuries has been entrusted with the absolute values of human life, so, in this time of change, when some event shatters medical complacency, if that trust is to be safeguarded, the event must be closely examined. Such an event took place in Cape Town in December 1967 (Barnard, 1967).

The first heart transplant (Hardy et al., 1964)largely because the donor was an ape-was greeted by the medical world with considerable repugnance. Likewise when the news from Cape Town came, a similar repugnance gripped many. However, for Louis Washkansky the first postoperative seconds turned to weeks and soul-searching began. Such questions as 'is it feasible?', 'is it right?', 'has it passed beyond the purely experimental?', 'is it progress?', had to be answered. They could not be answered, but when 6 months later Dr Blaiberg was apparently well one felt that the answer to these questions was 'yes'.

In short, cardiac transplantation seemed tenable. The work begun by Alexis Carrel (1905) had become reality. For the members of our group therefore, the first prerequisite, that of conviction, seemed to have been established.

The next step was to establish for outselves rigid standards of procedure. These standards related to the physical and mental state of the patient, the decision regarding surgery, and the duty to a donor.

\section{Physical state}

The patient, Richard Pye, aged 57 years, first experienced exertional angina in July 1963. In August 1963, he suffered an anterior myocardial infarct. In 1964, he developed congestive cardiac failure, but despite considerable restriction in effort tolerance he continued as a maintenance foreman on the Sydney Harbour Bridge until 1966. From then on he was a cardiac invalid, and was admitted on numerous occasions to the Repatriation General Hospital, Concord, for treatment of intractable congestive cardiac failure. In the 6 weeks prior to transplantation he had great difficulty walking the shortest distance, and was constantly troubled by nocturnal dyspnoea. He frequently gave the impression that he would not survive the night.

The clinical signs progressively worsened during the weeks preceding surgery. There was marked peripheral cyanosis extending to the knees and elbows, a raised jugular venous pressure reaching a maximum of $15 \mathrm{~cm}$ above the sternal angle, ankle and sacral oedema, hepatomegaly, cardiomegaly, gallop rhythm, a soft apical pansystolic murmur and bilateral basal crepitations. The blood pressure was $100 / 70 \mathrm{mmHg}$ and sinus rhythm was present. The electrocardiogram showed evidence of an old anterior myocardial infarct with right bundle branch block. The chest X-ray showed cardiomegaly with congestive changes in the lungs. The blood urea was $70 \mathrm{mg} / 100 \mathrm{ml}$. Cardiac catheterization revealed marked elevation of the pressures in the pulmonary artery and right heart secondary to a left ventricular end-diastolic pressure of $33 \mathrm{mmHg}$. The cardiac index was $1.81 / \mathrm{m}^{2} / \mathrm{min}$ rising to $2.41 / \mathrm{m}^{2} / \mathrm{min}$ on exercise. Cineangiography showed a grossly dilated, poorly contracting left ventricle. There was diffuse obstructive disease of all three major coronary artery branches.

Treatment before surgery varied almost daily, but the following list covers an average day: digoxin $0.5 \mathrm{mg}$, frusemide $200 \mathrm{mg}$, ethacrynic acid $200 \mathrm{mg}$, spironolactone $100 \mathrm{mg}$, potassium supplements, warfarin and periodic mannitol diureses when oedema became gross. Frequent additional intramuscular frusemide was required.

In summary, the patient had gross and irreversible myocardial disease with intractable congestive cardiac failure. His outlook seemed hopeless. He did not appear to have serious disease of other organs (Gibson et al., 1969).

The question of both arterial implant and left ventricular resection was considered, but both were discarded as not being surgically feasible in view of the extensive myocardial fibrosis.

This case history illustrates the first standard, namely that the physical state of the heart was such 
that it had brought its owner to the end of the road and no form of therapy other than transplantation seemed feasible.

This assessment was borne out by the later examination of the resected heart of which the following is a brief description.

There was extensive antero-septal infarction of the left ventricle. The thickness of the wall diminished from $20 \mathrm{~mm}$ at the base to $2 \mathrm{~mm}$ at the apex. The left ventricular cavity was sac-like rather than aneurysmal. The muscle was largely replaced by dense collagen, apart from a narrow zone of surviving myocardium immediately beneath the endocardium. The endocardium was white and opaque, because of a marked degree of fibro-elastosis. The inter-ventricular septum was fibrosed and aneurysmal. There were scattered areas of fibrosis and endocardial fibro-elastosis in the right ventricle. The individual surviving myocardial fibres showed marked hypertrophy. The coronary arteries were the site of severe atherosclerosis, all three major vessels being occluded.

\section{Mental state}

Richard Pye was well acquainted with newspaper accounts of transplantation. Every aspect of a publication (Windsor, 1968) was discussed in detail with him. He accepted the experimental nature of the surgery, the slenderness of his chance, the small likelihood of lasting benefit, the duty to a donor, and the basic motives of a surgeon. He was equally tenacious of life and fearless of death. His iron will and determination to submit himself to transplantation in an effort to live without pulmonary oedema was proof of this, and as such was of more influence in the decision than the purely physical characteristics of his heart.

Formerly we felt that transplantation had no place for the moribund who lack the full use of their faculties, cannot appreciate the problem and have no insight. Richard Pye, whilst very near the end of the road, was not moribund. His mental attitude was the feature which marked the line of separation.

As of the present time we agree with Stinson (1969) that under certain circumstances, for example, in a young person moribund following a massive myocardial infarct, transplantation might be indicated, and that a close relative might well give permission for the procedure.

\section{Decision regarding surgery}

Most patients, unable to understand the technical considerations, explicitly trust their doctor and will consent to any proposal. Nearly always they accept the doctor's judgement as decisive. When Richard Pye entered unreservedly into the proposal a com- mittee of senior consultants, not exclusively cardiologists, but all long experienced in dealing with men, was appointed by the Repatriation Commission. This committee had little difficulty in deciding that transplant was indicated. This approval of impartial and distinguished colleagues was of great help to the morale of the surgeon.

When the decision had been made the patient confirmed his apparent fortitude by the uncomplaining composure with which he waited until the day of surgery, 23 October 1968.

\section{The donor}

The absence of cardio-respiratory function has been so deeply rooted in the minds of men as the criterion of death that now, when we are asked to certify death in the presence of cardio-respiratory function, discussion flares.

We are aware that cessation of cardio-respiratory function is rapidly followed by cessation of cerebral function. The reverse is likewise true except that by modern means cardio-respiratory function can be maintained in the presence of an irreversibly damaged brain (Crafoord, 1969). The heart will on occasion continue to beat for a variable time without respiratory assistance as autopsy reports on criminals judicially hanged have shown.

The discussion is related to the fact that many find it impossible to accept the difference between cardio-respiratory function regulated by the brain and cardio-respiratory function regulated artifically without connection with the brain.

It was this dilemma which the Declaration of Sydney (1968) endeavoured to solve when it stated, 'It is the moment of irreversibility of the process leading to death which must be determined, rather than the moment of death'. The latter it is pointed out defies exact definition, it varies organ to organ, cell to cell, so that it is impossible to say precisely when human life becomes extinct.

This infers that irreversibility can be established before the cessation of cardio-respiratory function. Many of the groups undertaking cardiac transplantation have accepted this and maintained the donor in an optimum condition by artificial means with the heart beating until the time of removal. Exactly the same thing is achieved by maintaining optimum conditions until the time for removal arrives, then turning off the respirator and waiting until the heart has stopped before proceeding.

In our case it had been decided that no move would be made until the donor heart had ceased to beat. This was respected and the recipient was not anaesthetized until after the donor's chest had been opened, his heart had been restarted and he had been placed on supportive cardio-pulmonary bypass. This respect for the time-honoured criterion added 
greatly to the difficulty and length of the operation. Irreversibility had occurred many hours before the donor's heart had ceased to beat.

The debate then, is whether, after a competent independent team has decided that irreversibility has occurred, we are justified in crossing the Rubicon and carrying out synchronous surgery. I think we are, for when irreversibility has been established that is the moment when responsibility to the donor is superceded by responsiblity to the recipient. To do otherwise is not a matter of prolonging the donor's life, it is merely a matter of prolonging the process of his dying.

The law and public rightly demand that such a decision be made only by a wise, competent and independent team, and that neither incompetence, laziness nor inexperience enter the decision. If this is adhered to, Dr Ramsay (1968) will be unable to repeat 'Death for the clinician is no longer death as the lawyer assumes it'.

Rightly many argue 'the donor might recover'. If there is any question that irreversibility has not occurred the independent team has shown neither wisdom nor competence.

\section{Operation}

Except in so far as that donor and recipient were in adjoining rooms, surgery was not synchronized. This could have proved fatal for the recipient, as he was not anaesthetized until the donor had been certified dead. The bulk of the recipient's heart and the dense vascular adhesions then made the excision more difficult than had been anticipated, with the result that $2 \mathrm{hr} 3 \mathrm{~min}$ elapsed after the death of the donor before the heart was placed in the recipient's pericardial sac (Windsor, 1969).

Unquestionably if surgery could be carried out as a definitive and synchronized procedure, every effort being made to maintain the haemodynamics of the donor as near normal as possible, the chances of success would be greater. It is apparent that this is being done in most centres (de Bakey et al., 1969) whether or not the donor heart is kept beating until removal or the respirator turned off at a time to synchronize with readiness of the recipient for transplant. It would be of great help towards the solution of this vital issue if those who have carried out transplants stated truthfully and explicitly in their writings just exactly what happened instead of such statements as 'after the death of the donor'.

The surgical technique followed the well-defined methods pioneered by Shumway, Lower \& Stofar (1959) and Lower et al. (1960, 1961, 1962). The inferior vena cava was cannulated through the femoral vein. We now believe that groin incisions should be avoided and that the most satisfactory way to induce cardio-pulmonary bypass is to cannulate the superior vena cava through the internal jugular vein and the inferior vena cava through the superior vena cava and to return the arterial blood to the ascending aorta.

The donor heart was intermittently perfused with blood at $30^{\circ} \mathrm{C}$ from the pump oxygenator via the innominate artery. That some form of myocardial protection is necessary for the donor heart was the consensus of opinion at the Second World Symposium on Heart Transplantation (Montreal, 1969). That transfer can be accomplished at normothermia without myocardial protection is unquestionable (Cooley, 1969), but it is possible that the incidence of apparent acute rejection would be lowered by the use of myocardial protection. Total donor body hypothermia and subsequent immersion of the heart in saline at $4^{\circ} \mathrm{C}$ or coronary perfusion with blood at $30^{\circ} \mathrm{C}$ are the methods of choice. Personal preference is for the latter.

The dense vascular adhesions contributed to excessive post-bypass bleeding, difficulty in drainage of the pericardial sac and the later development of an intra-pericardial space which became infected and led to the death of the patient on the forty-fifth post-operative day.

\section{Pathology}

It seems appropriate at this stage to include a brief description of the microscopic features of the donor heart.

The transplanted heart at autopsy showed none of the usual macroscopic signs of rejection, namely enlargement, oedema, mottling or subendocardial haemorrhage. Microscopically there were areas of infarction in both donor and recipient atria. In the ventricles there were areas of myocytolysis and collapse fibrosis, whilst occasional capillaries contained thrombi. In the areas of myocytolysis the myofibrils had disappeared leaving empty intact endomyseal sheaths among which were small numbers of macrophages containing lipofuscin pigment (Ralston, 1969).

The features of rejection were few but it was felt that both the myocytolysis and capillary thrombi indicated minor rejection in a patient on immunosuppressive therapy. The intermittent anoxia to which the donor heart was subjected between its removal from the donor and the re-establishment of circulation in the recipient might have been responsible for the foci of collapse fibrosis but is unlikely to have caused the myocytolysis present at autopsy.

The features of acute rejection, namely wide separation of myocardial fibres with lymphocytes, plasma cells, macrophages and leucocytes, degeneration of the media and fragmentation of the endothelium were not seen.

In some but not all patients who have lived an 
appreciable time following transplant, diffuse thickening and atheroma of the intima of the large coronary vessels has been found. Lower (1969) has stressed that to establish why this happens, why some escape, and how it can be prevented is vital for future success.

\section{The postoperative course}

Death of the patient occurred on the forty-fifth day but was not related to cardiac function of which there had been progressive clinical improvement from the time of transplant. Cardiac output and peripheral circulation remained satisfactory from the early postoperative days until the day of death except as detailed below. Nocturnal dyspnoea and orthopnoea, severe before surgery were not present after transplantation.

\section{Rejection}

The recipient's red blood cell group was A1 Rh positive. The donor was $\mathrm{A} 2 \mathrm{Rh}$ positive and his serum contained no anti-A antibodies. The histocompatibility antigens showed two incompatible matches and four non-identical matches. For this reason vigorous immunosuppressive treatment was given (Biggs, 1969).

Rejection was diagnosed and treated on three occasions on the eighth, fifteenth and thirty-first days. The first of these episodes was characterized by mild right heart failure and a gradual but small decline in the $R$ wave voltages in Lead II. The second episode was associated with marked right ventricular failure, a right ventricular diastolic gallop, atrial arrhythmias and peripheral cyanosis. The chest X-ray showed cardiomegaly. The third episode was associated with atrial arrhythmias, a pericardial friction rub and mild right ventricular failure. No significant $R$ wave voltage changes occurred during the last two episodes (Breslin et al., 1969). In none of the episodes were there significant changes in pulse rate, blood pressure or pulse pressure. Treatment was restricted to an increase in steroid therapy using methyl prednisolone $(600 \mathrm{mg} /$ day $)$ for 3 days in addition to the maintenance dose of prednisone $(1.2 \mathrm{mg} / \mathrm{kg})$ and azathioprine $(2.5 \mathrm{mg} / \mathrm{kg})$.

A critical appraisal of these three episodes throws doubt on whether in fact they were manifestations of rejection. This is particularly so with the second episode for the signs of rejection disappeared following re-operation and evacuation of a pericardial collection containing $700 \mathrm{ml}$ of sterile blood-stained fluid, thereby suggesting that tamponade was responsible.

The erythrocyte sedimentation rate, enzyme studies, $\mathbf{R}$ wave voltages and the presence of atrial arrhythmias were not particularly helpful. At autopsy the evidence for rejection was minimal.
We were influenced in our management of these episodes by the work of Stinson and Shumway, particularly in reference to the electrocardiographic changes. They consider that decline in voltages in Lead II is the most significant change, but that supraventricular arrhythmias, conduction disturbances and shifts in electrical axis are occasionally present (Stinson et al., 1969).

Two features of the rejection process are relevant to the clinical management. The first of these, emphasized by Lower (1969) is the sequence of platelet deposition, intimal proliferation, thrombosis, organization and later fibrosis occurring in the vessels of the transplanted organ. This has led Stinson and his colleagues to incorporate systemic treatment with heparin as part of the management of acute rejection (Stinson et al., 1969). Kahn (1969) used anticoagulants as a routine in the postoperative management of three successful transplants.

The second emphasized by Stinson et al. (1969) postulates significant regional differences in apparent immunologic response in the heart, especially the denser cellular infiltrate contained within Purkinje's fibres of the common bundle of His. This they consider might explain some of the early electrocardiographic features of acute rejection.

\section{Infection}

A tracheostomy was carried out at the termination of the operation. On the sixth postoperative day bilateral pneumonia was present. This was considered to be due to Serratia marcescens, which was cultured from the purulent tracheo-bronchial aspirate. This was successfully managed with kanamycin $(1 \mathrm{~g}$ per day) for 7 days. Staphylococcus albus and Klebsiella were cultured continuously from the respiratory secretions during the whole postoperative period, but they were not considered pathogenic. Pseudomonas aeruginosa (Pyocyanea) was frequently recovered from the sputum. A round lesion appeared in the chest X-ray during the fifth postoperative week. At autopsy this proved to be a lung abscess containing Pseudomonas aeruginosa and Aspergillus flavus. Ulcers subsequently proved to be due to herpes simplex appeared on the tongue in the sixth week (Burns, 1969).

A sterile pericardial effusion was evacuated and pericardial drainage instituted on the sixteenth postoperative day. A thin discharge from the pericardial drainage sites commenced soon after removal of the tubes. From this was cultured Serratia marcescens. As the patient remained well and the cardiac silhouette remained small, no active measures were taken until retrosternal pain became persistent. On the forty-second day a small pericardial space containing thin pus from which was cultured Serratia marcescens was evacuated. Death occurred suddenly 
on the forth-fifth day from haemorrhage of aortic origin.

Similar opportunistic organisms have caused the death of many patients on immunosuppressive therapy. Infection in the presence of immunosuppression remains a great problem. Progress is slow but light is appearing. Kahn (1969) described the successful treatment of Pseudomonas lung abscess and empyema in one of his transplants by means of colistin and human Pseudomonas immuneglobulin.

\section{Anaphylaxis}

Antilymphocyte globulin supplied by the Auckland, New Zealand, blood tranfusion service was used. A rising titre of antibodies to horse serum was demonstrated, as was a peripheral lymphopaenia. Later at autopsy depletion of the lymphoid cells in the spleen and abdominal lymph nodes was noted. It was necessary to suspend ALG at the end of the fifth week because of severe unpredictable anaphylaxis type reactions (Biggs, 1969).

Kashiwagi et al. (1968) reported anaphylactic reactions in $21 \%$ of patients receiving ALG. In $15 \%$ it was necessary to suspend the ALG.

At present the effectiveness of ALG is doubtful. There is no accurate method of standardization either in vivo or in vitro. There is no way of assessing the effectiveness of a batch. Kahn (1969) in three successful cardiac transplants did not use ALG. However, in this field, light is also beginning to appear. Moberg et al. (1969) have reported a technique for rapidly producing large volumes of ALG which is free of protein contamination, is given by the intravenous route and has been shown to be immunosuppressive in human beings.

\section{The future}

There has been increasing critical appraisal by both public and profession of cardiac transplantation. This is not surprising when one considers how the problem is hedged by innumerable apparently insoluble difficulties.

We formerly felt that only a recipient mentally adjusted to such an undertaking should be selected, but now believe that a young person dying from a massive myocardial infarct ought to be considered even though he be unable to make the decision himself. Granted resuscitation, supportive cardiopulmonary bypass, or an artificial heart prior to elective transplantation have still to be perfected, but progress in all is being made (Butner et al., 1969; Cooley, 1969).

It can be argued that prevention is the answer, but it is unrealistic to believe that prevention will be either immediate or complete. In twelve patients accepted for transplantation at Stanford, but for one reason or another not submitted to transplantation, the mean survival time was 1 month (Shumway, 1969). Therapy must continue to take its place beside prevention.

Donor selection in terms of meeting the demand and the immemorial conflict between conservatism and progress will long remain a problem. Our attitude has been clearly stated.

The disadvantages of non-selective immunosuppression, namely reduced capacity to heal, lowered resistance to bacterial and viral infection, bone depression, fluid retention, cellular damage, liver damage and even tumour formation are such as to lead some to advocate waiting for complete mutual histocompatibility. Here again it is unlikely that perfect tissue matching will become a reality in anything like the near future, whereas the management of allograft rejection improves from day to day with numerous efforts aimed at inducing a state of specific immunological tolerance. Steroids, antimetabolites, immunosuppression and local radiotherapy (Graham et al., 1969) are all improving and with that comes increasing allograft survival.

The goals are ambitious. Scientific improbabilities are there to be overcome and should not be looked upon as harbingers of defeat. Lord Rutherford 35 years ago, when asked if he foresaw any practical application of atomic energy, replied 'Never' (Medcom, 1969).

There seems no reason why we should not continue if rigid standards are followed; to do otherwise will merely hamper progress. If we accept only the strictest criteria in respect of recipient and donor selection, we shall do fewer transplants, but we shall help more people and we shall certainly go a long way towards establishing the confidence of both public and profession in our efforts.

\section{Acknowledgment}

Sincere thanks to the Editor, The Medical Journal of Australia, for permission to quote liberally from the issue of 29 March 1969.

\section{References}

BARNARD, C.N. (1967) The operation. A human cardiac transplant. South African Medical Journal, 41, 1271.

BigGS, J.C. (1969) A heart transplantation: 8 immunological and haematological aspects. Medical Journal of Australia, i, 681.

Breslin, A.B.X., Morgan, J.J., Seldon, W.A. \& Shanahan, M.X. (1969) A heart transplantation: 5 post operative medical aspects. Medical Journal of Australia, i, 673.

BURNS, M.W. (1969) A heart transplantation: 7 microbiology and antibiotic chemotherapy. Medical Journal of Australia. i, 679.

Butner, A.N., Krakauer, J.S., Rosenbaum, A., TuønneLAND, S., Sherman, J.L., Dresdale, D.T. \& KaNtrowitz, A. (1969) Clinical trial of phase shift balloon pumping in 
cardiogenic shock. Results in 29 patients. 55th Annual Clinical Congress American College of Surgeons, Surgical Forum, 20, 199.

Carrel, A. \& Guthrie, C.C. (1905) The transplantation of veins and organs. American Medicine, 10, 1101.

Cooley, D.A., Hallman, G.L., Bloodwell, R.D., Nora, J.J. \& Leachman, R.D. (1968) Human heart transplantation: experience with 12 cases. American Journal of Cardiology, 22, 804.

Cooley, D.A. (1969) Report on the use of an implantable artificial heart. Second World Symposium on Heart Transplantation, Montreal.

Crafoord, C.C. (1969) Cerebral death and the transplantation era. Diseases of the Chest, $55,141$.

De BakeY, M.E., Diethrich, E.B., Glick, G., Noon, G.P., BUTLER, W.T., RosSEN, R.D., LidDICOAT, J.E. \& BROOKS, D.K. (1969) Human cardiac transplantation; clinical experience. Journal of Thoracic and Cardiovascular Surgery, $58,303$.

Declaration of Sydney (1968) British Medical Journal, 4, 449.

Gibson, H., Seldon, W.A. \& Windsor, H.M. (1969) A heart transplantation. 1. Clinical features. Medical Journal of Australia, i, 663.

Graham, W.H., Childs, J.W., Weymouth, R.J., KoseK, J.C., LIM, F. \& Lower, R.R. (1969) The reversibility of dog cardiac allograft rejection. 55th Annual Clinical Congress American College of Surgeons. Surgical Forum, 20, 145.

Hardy, J.D., Chavez, C.M., Kurrus, F.D., Neely, W.A., Eraslan, S., Turner, M.D., Fabian, L.W. \& Labecki, T.D. (1964) Heart transplantation in man. Developmental studies and report of a case. Journal of the American Medical Association, 188, 1132.

KAHN, D.R. (1969) Second World Symposium on Heart Transplantation, Montreal.

Kashiwagi, N., Brantigan, G.O., Brettschneider, L., GROTH, D.F. \& STARZL, T.E. (1968) Clinical reactions and serological changes after the administration of heterologus antilymphocyte globulin to human recipients of renal homografts. Annals of Internal Medicine, 68, 275.

LOWER, R.R. \& SHUMWAY, N.E. (1960) Studies of orthotopic homotransplantations of the canine heart. Surgical Forum, 11, 18.
Lower, R.R., Stofar, R.C. \& Shumway, N.E. (1961) Homovital transplantation of the heart. Journal of Thoracic and Cardiovascular Surgerv, 41, 196.

Lower, R.R., Stofar, R.C., Hurley, E.J., Dong, E.J. Cohn, R.B. \& Shumway, H.E. (1962) Successful homotransplantation of the canine heart after anoxic preservation for seven hours. American Journal of Surgery, 104, 302.

Lower, R.R. (1969) Second World Symposium on Heart Transplantation, Montreal.

LOWER, R.R. (1969) Panel Discussion. 55th Annual Clinical Congress American College of Surgeons.

Medcom (1969) Transplant. Library of Congress Catalogue Number 68-58937.

Moberg, A.W., Gewurz, H., Simmons, R.L., Gunnarsson, A., Merkel, F. \& Najarian, J.S. (1969) A new efficient method for preparation of immunoelectrophoretically pure horse antihuman antilymphoblast globulin. 55th Annual Clinical Congress American College of Surgeons. Surgical Forum, 20, 261.

Ralston, M. (1969) A heart transplantation: 7 pathological aspects. Medical Journal of Australia, i, 676.

Ramsay, I. Anglican Bishop of Durham (1968) Guardian, 7 May. Quoted in Herder correspondence, Vol. 5, p. 240.

Second World Symposium on Heart Transplantation (1969) Montreal.

Shumway, N.E., Lower, R.R. \& Stofar, R.C. (1959) Selective hypothermia of the heart in anoxic cardiac arrest. Surgery, Gynaecology and Obstetrics, 109, 750.

Shumway, N.E. (1969) Lecture Outlines, 55th Annual Clinical Congress, American College of Surgeons, p. 82.

Stinson, E.B., Dong, E., Bieber, C.P., Schroder, J.S. \& ShumwaY, N.E. (1969) Cardiac transplantation in man: 1. Early rejection. Journal of the American Medical Association, 207, 2233.

Stinson, E.B. (1969) Personal communication.

WINDSOR, H.M. (1968) Heart transplantation or keeping both feet on the ground. Medical Journal of Australia, $i$, 869 (Letter).

WindSOR, H.M. (1969) A heart transplantation: 3. Surgical and post operative surgical aspects. Medical Journal of Australia, i, 666. 